\title{
Magnetic Hyperfine Structure and Core Polarization in the Excited States of Lithium*
}

\author{
William A. Goddard, III ${ }^{\dagger}$ \\ Alfred A. Noyes Laboratory of Chemical Physics, $\$$ \\ California Institute of Technology, Pasadena, California 91109 \\ (Received 25 June 1968)
}

\begin{abstract}
The magnetic hyperfine splitting constants, $a_{J}$, from theoretical calculations on the $3^{2} S$, $2^{2} P, 3^{2} P$, and $3^{2} D$ excited states of the $\mathrm{Li}$ atom are reported. The wave functions were calculated using the author's GF method, which corresponds to optimizing the orbitals of a Slater determinant after spin projection. Thus the wave functions include core polarization, but no appreciable correlation. For the $2^{2} P$ state we calculate $a_{1 / 2}=0.2206$ a.u. $(45.74 \mathrm{Mc} /$ sec for ${ }^{7} \mathrm{Li}$ ) which is in good agreement with the experimental value, $0.2227 \pm 0.0017$ a.u. $\left(46.17 \pm 0.35 \mathrm{Mc} / \mathrm{sec}\right.$ for $\left.{ }^{7} \mathrm{Li}\right)$, and the value from configuration interaction calculations, 0.2206 . Thus for the $\mathrm{Li}$ atom core polarization accounts for most of the error in the HartreeFock values of $a_{J}$. These calculations yield $\left\langle 1 / \gamma^{3}\right\rangle$ and spin density, $Q(0)$, in agreement with other accurate theoretical calculations, and in disagreement with the values found using level crossing experiments, indicating that the interpretation of the level crossing experiments in terms of $\left\langle 1 / \gamma^{3}\right\rangle$ and $Q(0)$ may not be correct. It is found that for both the unrestricted Hartree-Fock and GF methods the use of a different Hamiltonian for each electron leads to virtual orbitals which are good approximations to the actual orbitals of excited states.
\end{abstract}

\section{INTRODUCTION}

The Fermi contact portion of the magnetic hyperfine interaction is proportional to the spin density at the nucleus, $Q(0)$. The Hartree-Fock (HF) wave function for an atom such as $\mathrm{Li}^{2} P\left(1 s^{2} 2 p\right)$, $\mathrm{N}{ }^{4} S\left(1 s^{2} 2 s^{2} 2 p^{3}\right)$, or $\mathrm{O}^{3} P\left(1 s^{2} 2 s^{2} 2 p^{4}\right)$ has all $s$ orbitals doubly occupied and hence leads to a zero Fermi contact interaction. However, the experimental results show that the Fermi contact term is not zero in these atoms $\mathrm{s}^{1,2}$ and thus that the spin density at the nucleus is nonzero. This clearly indicates that to study such interactions we must improve upon the Hartree-Fock wave function. One approach has been to use configuration interaction (CI), ${ }^{3,4}$ where many Slater determinants are formed from a large set of orbitals, and the determinants are combined such as to minimize the total energy. If this is carried out for a complete set of orbitals, we must get the exact wave function, and thus for a sufficiently large basis set we should be able to get arbitrarily good energies and spin densities and correctly account for the magnetic hyperfine interactions.

Another approach has been to retain the Slater determinant form of the wave function, but to remove some of the other restrictions of the HartreeFock method. In particular if we allow the $1 s$ orbitals of the $\mathrm{Li}^{2} P$ wave function to be different for different spins, there will be a net spin density at the nucleus, $\left|\phi_{1 a}(0)\right|^{2}-\left|\phi_{1 b}(0)\right|^{2}$, where $\phi_{1 a}$ is associated with $\alpha$ spin and $\phi_{1 b}$ with $\beta$ spin. This procedure, which is called the unrestricted Hartree-Fock (UHF) method, ${ }^{5}$ has the disadvantage ${ }^{6}$ that the many electron wave function is not an eigenfunction of $\hat{S}^{2}$. Since the property we are considering depends on spin, we are led to consider the spin projected UHF (PUHF) wave function in which we delete the parts of the UHF wave function having the wrong spin. However, in this case we are using the orbitals optimized for the unpro- jected (UHF) function, whereas we really should use the orbitals optimized for the projected function. This latter approach in which the orbitals are optimized after spin projection is equivalent to the GF method mor $^{7-9}$ for electronic wave functions. That is the GF wave function is the best possible spin projected Slater determinant wave function. ${ }^{7}$

Since the GF wave function is based on spin projection of a single Slater determinant, it will not coincide with the exact wave function and will not yield the spin density exactly. However, it does take into account the direct spin polarization of the core (we will call this core polarization) caused by the unpaired spins of the valence electrons. Any difference between the GF value of $Q(0)$ and the exact value could be accounted for by mixing in sufficient other spin projected determinants; such effects we will simply ascribe to correlation. Thus we distinguish the simple exchange correlation involved in core polarization and accounted for by GF wave functions from the other kinds of correlation.

We will see that just as for the $\mathrm{Li} 2{ }^{2} S$ ground state $^{8}$ the GF wave function also accounts for the hyperfine structure of the $\mathrm{Li} 2^{2} P$ state, and thus corrections beyond direct core polarization are not too important for $\mathrm{Li}$.

\section{CALCULATIONS}

The $2{ }^{2} S, 3{ }^{2} S, 2{ }^{2} P, 3{ }^{2} P$, and $3{ }^{2} D$ states of $\mathrm{Li}$ were considered, and UHF, PUHF, and GF calcutions were carried out for each state. Since HF wave functions for these states have been published by Weiss, ${ }^{4}$ we used basis sets based upon his calculations. These basis sets of Slater orbitals ${ }^{10}$ were selected so as to satisfy the cusp condition at the nucleus [this seems to be important for reliable values of $Q(0)$, especially for larger atoms such as $N$ ]. It was established that for $\mathrm{Li}$ the optimum orbital exponents for all three types of wave 
functions (HF, UHF, and GF) are essentially the same. Four of the $n s$ orbitals were primarily used by the core electrons and had been taken to have the same orbital exponents in all of Weiss's calculations. We reoptimized these orbitals for the $2{ }^{2} P$ state and found that the new orbital exponents also led to lower energies and better virial ratios for the other states. Thus we changed Weiss's basis sets by using these reoptimized (for the $2{ }^{2} P$ state) exponents for the core orbitals.

A self-consistent-field calculation (e.g. , HF, UHF, or GF) for the second-lowest state of some symmetry [e.g. , $3^{2} P\left(1 s^{2} 3 p\right)$ ] is straightforward and leads to an upper bound on the exact energy of the excited state (see Appendix C). However, for the $3^{2} S$ and $3^{2} P$ states of $\mathrm{Li}$ the solutions of the usual form of the UHF and GF equations diverged and oscillated. This required using the form of the self-consistent-field equations derived directly from the variational theorem rather than the usual form of the equations in which self-repulsion terms are added. This is discussed later.

\section{CORE POLARIZATION}

The calculated hyperfine splitting constants are given in Table I (see Appendix $\mathrm{B}$ for the relationships between $a_{J}$ and $Q(0)$ and $\left\langle 1 / r^{3}\right\rangle$ ). As occurred for the $2{ }^{2} S$ state, ${ }^{7}$ the $a_{1 / 2}$ for $2^{2} P$ as calculated by both the GF and $\mathrm{CI}^{11}$ methods is in good agreement with experiment. ${ }^{12}$ Since the GF results are close to experiment, we see that the main error in the

TABLE I. Hyperfine splitting constants and other properties for the ground and excited states of $\mathrm{Li}$ as obtained from GF and other wave functions. $E$ is the total energy, $V$ is the potential energy, $V / 2 E$ is the virial ratio, $\rho(0)$ is the electron density at the nucleus, and $Q(0)$ is the spin density at the nucleus. All quantities are in atomic units (the hartree is the unit of energy).

\begin{tabular}{|c|c|c|c|c|c|c|c|c|}
\hline & $E$ & $V / 2 E$ & $\rho(0)$ & $\left\langle\sum r^{2}\right\rangle$ & $Q(0)$ & $\left\langle 1 / r^{3}\right\rangle$ & ${ }^{a_{L-\frac{1}{2}}}$ & ${ }^{\mathrm{a}} L+\frac{1}{2}$ \\
\hline $2^{2} S \mathrm{HF}$ & -7.432725 & 1.000002 & 13.8160 & 18.638 & 0.1667 & $\ldots$ & $\therefore$ & 1.396 \\
\hline UHF & -7.432749 & 1.000000 & 13.8159 & 18.630 & 0.2248 & $\ldots$ & $\ldots$ & 1.883 \\
\hline PUHF & -7.432767 & 0.999999 & 13.8159 & 18.630 & 0.1866 & $\ldots$ & $\ldots$ & 1.563 \\
\hline GF & -7.432813 & 1.000000 & 13.8159 & 18.609 & 0.2406 & $\ldots$ & $\ldots$ & 2.016 \\
\hline $\mathrm{CI}$ & $-7.4779^{\mathrm{a}}$ & & & & $0.2249 \mathrm{~b}$ & $\ldots$ & $\ldots$ & 1.884 \\
\hline EXP & $-7.47807^{\mathrm{c}}$ & & & & $0.2313^{\mathrm{d}}$ & $\ldots$ & $\ldots$ & 1.937 \\
\hline $3^{2} S$ HF & -7.310210 & 0.999994 & 13.7080 & 119.480 & 0.03864 & $\ldots$ & .. & 0.3237 \\
\hline UHF & -7.310212 & 1.000002 & 13.7067 & 119.470 & 0.05253 & $\ldots$ & $\ldots$ & 0.4401 \\
\hline PUHF & -7.310213 & 1.000000 & 13.7067 & 119.469 & 0.04335 & $\ldots$ & $\ldots$ & 0.3632 \\
\hline$\underset{\text { EXP }}{\text { GF }}$ & $\begin{array}{l}-7.310216 \\
-7.35410^{\mathrm{c}}\end{array}$ & 1.000002 & 13.7067 & 119.447 & 0.05622 & $\ldots$ & $\ldots$ & 0.4710 \\
\hline $2^{2} P \mathrm{HF}$ & -7.365069 & 0.999995 & 13.6534 & 28.716 & 0.00000 & 0.05848 & 0.1559 & +0.0311 \\
\hline UHF & -7.365076 & 1.000000 & 13.6535 & 28.709 & -0.01747 & 0.05852 & 0.2407 & -0.0176 \\
\hline PUHF & -7.365080 & 1.000000 & 13.6535 & 28.709 & -0.00582 & 0.05852 & 0.1722 & +0.0149 \\
\hline GF & -7.365091 & 1.000002 & 13.6534 & 28.692 & $-0.02304 f$ & 0.05861 & 0.2206 & -0.0331 \\
\hline CI & $-7.40838^{\mathrm{e}}$ & & & & $-0.02222^{f}$ & $\mathrm{~g}$ & 0.2206 & -0.0302 \\
\hline EXP & $-7.41016^{\mathrm{c}}$ & & & & & & $0.2227^{h}$ & i \\
\hline $3^{2} P \mathrm{HF}$ & -7.293186 & 0.999945 & 13.6660 & 172.536 & 0.00000 & 0.01759 & 0.04689 & +0.00937 \\
\hline UHF & -7.293187 & 0.999972 & 13.6661 & 172.530 & -0.005531 & 0.01760 & 0.06234 & -0.00607 \\
\hline PUHF & -7.293188 & 0.999972 & 13.6661 & 172.530 & -0.001843 & 0.017 .60 & 0.05204 & +0.00422 \\
\hline$\underset{\mathrm{EXP}}{\mathrm{GF}}$ & $\begin{array}{l}-7.293189 \\
-7.33715^{c}\end{array}$ & 0.999972 & 13.6661 & 172.511 & -0.007318 & 0.01759 & 0.06735 & -0.01106 \\
\hline $3^{2} D \mathrm{HF}$ & -7.291973 & 0.999936 & 13.6715 & 126.924 & 0.00000 & 0.004951 & 0.004948 & 0.001412 \\
\hline UHF & -7.291974 & 0.999966 & 13.6722 & 126.923 & -0.000036 & 0.004951 & 0.005008 & 0.001352 \\
\hline PUHF & -7.291974 & 0.999966 & 13.6722 & 126.923 & -0.000012 & 0.004951 & 0.004968 & 0.001392 \\
\hline GF & -7.291974 & 0.999966 & 13.6722 & 126.923 & -0.000053 & 0.004951 & 0.005037 & 0.001323 \\
\hline EXP & $-7.33552^{\mathrm{c}}$ & & & & & & & \\
\hline
\end{tabular}

$a_{E}$. A. Burke, Phys. Rev. 130, 1871 (1963).

bE. A. Burke, Phys. Rev. $\overline{135}$, A621 (1964).

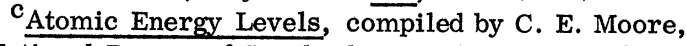
National Bureau of Standards Circular No. 467 (U. S. Government Printing Office, Washington; D. C. , 1947).

$\mathrm{d}_{\mathrm{P}}$. Kusch and H. Taub, Phys. Rev. 75, 1477 (1949); R. G. Schlect and D. W. McColm, Phys. Rev. 142, 11
(1966). The conversion factor from Mc/sec to a.u. is given in Appendix $B$.

eweiss, Ref. 4

$\mathrm{f}_{\text {Ardill and Stewart, Ref. } 18 .}$

$\mathrm{g}\left\langle 1 / r^{3}\right\rangle_{\text {orb }}=.05975,\left\langle 1 / r^{3}\right\rangle_{\text {dip }}=.05923$ Ref. 17.

$\mathrm{h}_{\text {Ritter, Ref. } 15 .}$

${ }^{i}$ See discussion in the Core Polarization section. 
HF calculations for the magnetic field at the nucleus, $\overrightarrow{\mathrm{H}}(0)$, is just the lack of core polarization. The error due to correlation is the difference between the GF and experimental values which we see is small.

The $a_{3 / 2}$ hfs constant for the $2{ }^{2} P$ state could not be measured directly. ${ }^{12}$ However, some level crossing experiments were carried out ${ }^{13,14}$ which led to effective values of $\left\langle 1 / r^{3}\right\rangle$ and $Q(0)$ that could be extrapolated to zero magnetic field in order to calculated $a_{3 / 2}$. The resulting value for $\left\langle 1 / r^{3}\right\rangle$ is $0.0645 \mathrm{a} . \mathrm{u}$. which differs from the $\mathrm{CI}$ value ${ }^{5,15}$ by about $8 \%$. The CI values of 0.0596 and 0.0594 for the $a_{1 / 2}$ and $a_{3 / 2}$ states are consistent with $\mathrm{HF}$, $\mathrm{UHF}$, PUHF, and GF values of 0.0585 to 0.0586 and should be expected to be within $1 \%$ of the exact value. Since the CI and GF values of $Q(0)$ and $\left\langle 1 / r^{3}\right\rangle$ are consistent with the experimental $a_{1 / 2}$, it may be that the interpretation of the experimental results in terms of $Q(0)$ and $\left\langle 1 / r^{3}\right\rangle$ are incorrect. ${ }^{15}$ In particular the external field, $\mathrm{H}$, must lead to perturbations in the core orbitals which depend on $\mathrm{H}$ and thus lead to effective values of $Q(0)$ and $\left\langle 1 / r^{3}\right\rangle$ which are different for each level and which depend on $\mathrm{H} .{ }^{14}$ Since the value of $a_{3 / 2}$ from the GF and CI calculations is a factor of two larger than that extrapolated from level crossing experiments, it may be that direct measurements at small field could determine which of these values is best even though it cannot ${ }^{12}$ give a precise value to $a_{3 / 2}$. (Note from Table I that the HF and PUHF wave functions lead to the wrong sign of $a_{3 / 2}$ for the Li $2^{2} P$ and $3{ }^{2} P$ states. )

We should note here that since the UHF wave function is not an eigenstate of $\hat{S}^{2}$ or $\hat{J}^{2}$, the UHF values for $a_{J}$ are somewhat suspect since $a_{J}$ is necessarily defined for some specific value of $J$. We have used the usual ${ }^{13,16}$ (apparently reliable) procedure of pretending that the UHF wave function has the same $J$ and $S$ that it would have if the core orbitals were identical.

\section{Perturbation Approximations}

Rather than calculate the effect of core polarization self-consistently as in the GF method, several perturbation approaches (starting with the HF wave function) have been suggested. ${ }^{17}, 18$ One interesting approach ${ }^{18}$ lets the core orbitals be perturbed by the nuclear magnetic moment via the Fermi contact term. By solving for the electronic energy for the perturbed wave functions, one gets directly the core polarization contribution to $a_{J}$. Since the orginal perturbation is due to the nuclear moment, the distortion of the core orbitals should be relatively independent of the electronic state. Thus the core polarization in molecules or metals can be calculated more easily. This approach leads to an approximation to the UHF wave function and for the $2 s$ and $2 p$ states of Li leads to core polarization contributions to $Q(0)$ of 0.0482 and -0.0154 , respectively, which are about $85 \%$ of the UHF values. Similarly, a perturbation approximation ${ }^{19}$ to the GF function for the $2^{2} S$ state yields a core polarization contribution to $Q(0)$ of $81 \%$ of the $\mathrm{GF}$ value. Thus, self-consistency is important for a reliable value of $Q(0)$.
Summary on Core Polarization

The term core polarization refers only to changes which occur upon improving the wave function within the independent particle interpretation, i.e., as in going from HF to UHF or HF to GF. The GF results given here show clearly the importance of core polarization and thus justify the concept both for polarization by $s$ and non- $s$ electrons. ${ }^{20}$ Regardless of how the remaining correlation energy is taken into account, it leads to a minor change in $Q(0)$. By comparing the GF and $\mathrm{HF}$ energies, we see that although the core polarization effects are crucially important for $Q(0)$, they have an almost negligible (but numerically significant) effect on the energy, which is the reason that some very good (low-energy) calculations have yielded rather poor spin densities. ${ }^{21}$

\section{CALCULATIONS ON EXCITED STATES}

In carrying out calculations on the $3{ }^{2} S$ and $3{ }^{2} P$ excited states of $\mathrm{Li}$, we observed some interesting features which would be expected to occur generally when performing calculations on excited states of the same symmetry as some lower state. In order to keep the discussion clear and simple, we will discuss the $3^{2} S$ state of $\mathrm{Li}$.

In the UHF method for $\mathrm{Li}$ we consider a wave function of the form

$$
a\left[\phi_{1 a^{(1)} \phi_{2 a}}{ }^{(2) \phi} 1 b^{(3) \alpha(1) \alpha(2) \beta(3)],}\right.
$$

and require that the orbitals be optimum, that is, yield a stationary energy. (In the above expression $\alpha$ is the antisymmetrizer, and $\alpha$ and $\beta$ are the spin-up and spin-down eigenfunctions of $\hat{s}_{z}$.) The result is a set of three equations

$$
\begin{aligned}
& H_{1 a} \phi_{1 a}=\epsilon_{1 a} \phi_{1 a}, \\
& H_{2 a} \phi_{2 a}=\epsilon_{2 a} \phi_{2 a}, \\
& H_{1 b} \phi_{1 b}=\epsilon_{1 b} \phi_{1 b},
\end{aligned}
$$

the self-consistent solutions of which are the optimum orbitals. In (1)

$$
\begin{aligned}
& H_{1 a}=h+\mathcal{J}_{2 a}-\mathcal{K}_{2 a}+\mathfrak{J}_{1 b}, \\
& H_{2 a}=h+\mathfrak{J}_{1 a}-\mathfrak{K}_{1 a}+\mathfrak{J}_{1 b}, \\
& H_{1 b}=h+\mathcal{J}_{1 a}+\mathfrak{J}_{2 a},
\end{aligned}
$$

where

$$
\begin{aligned}
& \mathcal{J}_{i}(1)=\int d x_{2} \phi_{i}{ }^{*(2)} \phi_{i}(2) / r_{12} \\
& \varkappa_{i}(1)=\int d x_{2} \phi_{i}{ }^{*(2)} \hat{P}_{12} \phi_{i}(2) / r_{12}
\end{aligned}
$$

are the Coulomb and exchange operators and $\hat{P}_{12}$ permutes electrons 1 and 2. However, 


$$
\left(\mathcal{J}_{i}-\varkappa_{i}\right) \phi_{i}=0
$$

So (2) are usually replaced by

$$
\begin{aligned}
& H_{A}=h+\left(\mathcal{J}_{1 a}-\mathcal{K}_{1 a}\right)+\left(\mathcal{J}_{2 a}-\mathcal{K}_{2 a}\right)+\mathcal{J}_{1 b}, \\
& H_{B}=h-\mathcal{J}_{1 a}-\mathscr{J}_{2 b}-\left(\mathcal{J}_{1 b}-\mathcal{K}_{1 b}\right),
\end{aligned}
$$

where (3) has the same self-consistent solutions as (2),

$$
\begin{aligned}
& H_{A} \phi_{1 a}=\epsilon_{1 a} \phi_{1 a}, \\
& H_{A} \phi_{2 a}=\epsilon_{2 a} \phi_{2 a}, \\
& H_{B} \phi_{1 b}=\epsilon_{1 b} \phi_{1 b} .
\end{aligned}
$$

Besides the self-consistent solutions (i.e., the ones used in the field terms), (2) and (3) have an infinite number of other solutions, called the virtual orbitals. Let us consider the ground state of $\mathrm{Li}, 2{ }^{2} S\left[(1 s)^{2}(2 s)\right]$. Then $1 a$ and $1 b$ correspond to $1 s$ orbitals and $2 a$ corresponds to the $2 s$ orbital. From (1) and (2) we have

$$
\begin{aligned}
& \epsilon_{2 a}=\left\langle\phi_{2 a}|h| \phi_{2 a}\right\rangle \\
& +\mathscr{J}_{1 a, 2 a}-\Re_{1 a, 2 a}+\mathscr{J}_{1 b, 2 a},
\end{aligned}
$$

where

$$
\begin{aligned}
& \mathcal{J}_{1 a, 2 a}=\left\langle\phi_{2 a}\left|\mathcal{J}_{2 a}\right| \phi_{1 a}\right\rangle
\end{aligned}
$$

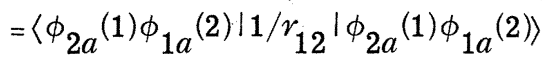

$$
\begin{aligned}
& \varkappa_{1 a, 2 a}=\left\langle\phi_{2 a}\left|\Re_{1 a}\right| \phi_{2 a}\right\rangle
\end{aligned}
$$

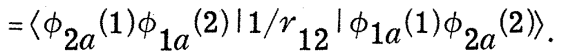

Hence (5) is just the difference in energy between the three-electron wave function $a\left(\phi_{1 a} \phi_{2 a} \phi_{1 b} \alpha \alpha \beta\right)$ and the two-electron wave function $a\left(\phi_{1 a} \phi_{1 b} \alpha \beta\right)$, and thus (5) is expected to be close to the ionization energy for the $2 s$ electron (Koopmans' theorem ${ }^{22}$ ). Now consider the solution of $\mathrm{H}_{2} a$ which has two nodes and is spherically symmetric (i.e., the $3 s$ virtual orbital), and call this orbital $\phi_{3 s^{2} a}$

$$
H_{2 a^{\phi}}{ }_{3 s}^{2 a}=\epsilon_{3 s}{ }^{2 a_{\phi}}{ }_{3 s} 2 a .
$$

Then from (2) and (6)

$\epsilon_{3 s}{ }^{2 a}=\left\langle\phi_{3 s}|h| \phi_{3 s}\right\rangle+\mathcal{J}_{1 a, 3 s}-K_{1 a, 3 s}+\mathfrak{J}_{1 b, 3 s},{ }^{(7)}$

which is just the difference between the energy of the three-electron wave function $a\left(\phi_{1 a} \phi_{3 s} 2 a_{\phi_{1 b}} \alpha \alpha \beta\right)$ and that of the two-electron wave function $a\left(\phi_{1 a} \phi_{1 b} \alpha \beta\right)$. Thus (7) is expected to close to the ionization energy for a $3 s$ electron, and the virtual solutions of $\mathrm{H}_{2 a}$ can be expected to be good approximations to the excited state orbitals of the $\mathrm{Li}$ atom.

Now consider the $3 s$ virtual solution of $H_{A}$

$$
H_{A} \phi_{3 s}^{A}=\epsilon_{3 s}{ }^{A} \phi_{3 s}{ }^{A} .
$$

From (3) this has an energy of

$$
\begin{aligned}
\epsilon_{3 s}{ }^{A} & =\left\langle\phi_{3 s}|h| \phi_{3 s}\right\rangle+\mathcal{J}_{1 a, 3 s}-\varkappa_{1 a, 3 s} \\
& +\mathcal{J}_{1 b, 3 s}+\mathscr{J}_{2 a, 3 s}-\varkappa_{2 a, 3 s} .
\end{aligned}
$$

But the ionization potential for the $3 s$ electron should be as in (7); thus $\epsilon_{3 s} A$ is too large by $\mathcal{J}_{2 a, 3 s}-\mathcal{K}_{2 a, 3 s}$, which is just the term arising from the addition of the self-Coulomb and exchange operators to $H_{2 a}$. Since $\mathcal{I}_{2 a}, 3 s>K_{2} a, 3 s$ the energies of the $A$-type virtual orbitals are all to high (in the case of the $\mathrm{Li}$ atom ground state, the virtual orbitals are all unbound), and the orbitals are too diffuse. This occurs because the virtual orbitals see an effective field due to $N$ other electrons rather than $N-1$ other electrons. (The $\phi_{3 s}{ }^{A}$ virtual orbital of $H_{A}$ is an approximation to the $\phi_{3 s}$ in $a\left(\phi_{1 a} \phi_{2 a} \phi_{3 s} \phi_{1 b} \alpha \alpha \alpha \beta\right)$, which is a wave function for the $3{ }^{3} S$ state of $\mathrm{Li}^{-}$, probably an unstable state.)

To illustrate these points, both (1) and (4) were solved for the ground state of Li using a basis set of 15 basis functions. The resulting eigenvalue spectra are given in Table II, where they are compared with the experimental and self-consistentfield values for the excited states. We see that the virtual solutions of $H_{2 a}$ are comparable to the self-consistent solutions for the excited states, whereas this is not at all true for the virtual solutions of $H_{A}$. The errors in the higher eigenvalues of $\mathrm{H}_{2} a$ are mainly due to the incompleteness of the basis set. If a complete basis set had been used, we probably would have obtained good values for the energies of all observed $n s, n p, n d$, and $n f$ states of Li. Thus if one wishes to study a series of rydberg states of an atom or molecule without doing each state self-consistently, the HF or UHF equations analogous to (1) should be used, rather than those analogous to (4). Actually, the core orbitals can be solved for with (3) as long as the orbital to be excited is solved for with the $H_{i}$ from (2).

If we wish to solve self-consistently for the $(1 s)^{2}$ (3s) ${ }^{2} S$ excited state of $\mathrm{Li}$, we just solve (1) where the $\phi_{2 a} a$ in $\mathcal{J}_{2 a} a$ and $\kappa_{2} a$ is taken to be the third lowest $s$ solution to $H_{2} a$ rather than the second lowest. As would be expected, this process works well, and the wave functions converge as well as for the ground state. We could also use the same process with (4) where we would take the first and third solutions of $H_{A}$ to be occupied. However, in this case the second and third $s$-type solutions are nearly degenerate, and the iteration process diverges (this problem also occurs for the $3^{2} P$ state of $\mathrm{Li}$ ). Thus we were forced to use (1) for 
TABLE $\Pi$. The energies for the $2 s$ orbitals and the virtual orbitals of Li from UHF and GF calculations (the basis set was based on the effective quantum numbers, $n^{*}$, from the term values of the lower $s$ and $p$ states. We used $1 s$, $2 s=2.7 ; 2 s, 3 s=0.63 ; 3 s, 4 s=0.39 ; 4 s, 5 s=0.28 ; 5 s=0.22 ; 2 p, 3 p=0.51 ; 3 p, 4 p=0.34 ; 4 p, 5 p=0.25$ where the Slater orbital is represented by $n l$ and the number is the orbital exponent) on the ground state, ${ }^{2} S\left[(1 s)^{2}(2 s)\right]$. Energies are in hartrees.

\begin{tabular}{|c|c|c|c|c|c|c|}
\hline \multirow[b]{2}{*}{ State } & \multicolumn{2}{|c|}{ UHF } & \multicolumn{2}{|c|}{$\mathrm{GF}$} & \multirow[b]{2}{*}{$\mathrm{SCF}^{\mathrm{a}}$} & \multirow[b]{2}{*}{ Experimental } \\
\hline & $\begin{array}{c}\quad \mathrm{H}_{2 a} \\
\text { Different } \\
\text { Hamiltonian }\end{array}$ & $\begin{array}{c}H_{A} \\
\text { Same }^{\mathrm{d}} \\
\text { Hamiltonian }\end{array}$ & $\begin{array}{c}H_{2 a} \\
\text { Different }^{\mathrm{c}} \\
\text { Hamiltonian }\end{array}$ & $\begin{array}{c}{ }_{A}^{H} \\
\text { Same } \\
\text { Hamiltonian }\end{array}$ & & \\
\hline $2 s$ & -0.1961 & -0.1961 & -0.1963 & -0.1963 & -0.1963 & -0.1982 \\
\hline $3 s$ & -0.0738 & +0.0017 & -0.0738 & -0.0152 & -0.0738 & -0.0742 \\
\hline $4 s$ & -0.0385 & +0.0077 & -0.0385 & -0.0060 & $\ldots$ & -0.0386 \\
\hline $5 s$ & -0.0236 & +0.0214 & -0.0236 & +0.0033 & $\ldots$ & -0.0236 \\
\hline $6 s$ & -0.0123 & +0.0548 & -0.0123 & +0.0289 & $\ldots$ & -0.0159 \\
\hline $2 p$ & -0.1283 & +0.0036 & -0.1283 & -0.0273 & -0.1287 & -0.1302 \\
\hline $3 p$ & -0.0567 & +0.0106 & -0.0567 & -0.0084 & -0.0568 & -0.0572 \\
\hline $4 p$ & -0.0317 & +0.0230 & -0.0317 & +0.0005 & $\ldots$ & -0.0320 \\
\hline $5 p$ & -0.0193 & +0.0516 & -0.0193 & +0.0221 & $\ldots$ & -0.0204 \\
\hline
\end{tabular}

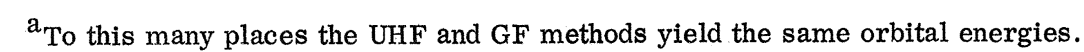

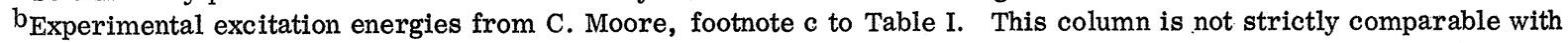
the other columns because of differences in the core states and correlation energy for the various states. However, these differences are small for the excited states.

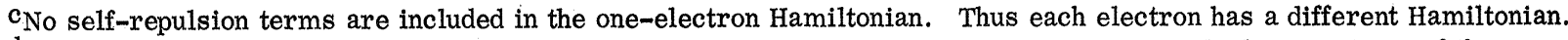

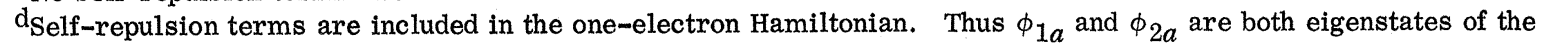
same Hamiltonian.

calculations on these excited states of $\mathrm{Li}$.

All these results are exactly the same in the GF case. The wave function is taken as

$$
G_{f}\left(\phi_{1 a^{\phi}}{ }_{2 a^{\phi}}{ }_{1 b} \alpha \alpha \beta\right)
$$

and the equations for the optimum orbitals are as in (1), ${ }^{7,9}$ where the forms of $H_{1} a, H_{2} a$, and $H_{1 b}$ are more complicated than (2). For example, after making a Roothaan expansion of the orbitals in terms of basis functions $\left\{X_{\mu}\right\}$ the matrix equivalent of $H_{2 a}$ has the for m ${ }^{7,9,23}$

$$
\begin{aligned}
H_{\mu \nu}^{2 a}= & \left(1+\frac{1}{2}\langle 1 a \mid 1 b\rangle^{2}\right)\langle\mu|h| \nu\rangle \\
& +\frac{1}{2}(\langle\mu|h| 1 b\rangle-\langle\mu|h| 1 a\rangle\langle 1 a \mid 1 b\rangle)\langle 1 b \mid \nu\rangle \\
& +\frac{1}{2}\langle\mu \mid 1 b\rangle(\langle 1 b|h| \nu\rangle-\langle 1 a|h| \nu\rangle\langle 1 b \mid 1 a\rangle) \\
& +\frac{1}{2}\langle\mu \mid 1 b\rangle\langle 1 b \mid \nu\rangle(\langle 1 a|h| 1 a\rangle-E) \\
& +(\mu \nu \mid 1 a 1 a)+(\mu \nu \mid 1 b 1 b)+(\mu \nu \mid 1 a 1 b)\langle 1 a \mid 1 b\rangle \\
& -(\mu 1 a \mid \nu 1 a)+\frac{1}{2}(\mu 1 b \mid \nu 1 b) \\
& -\frac{1}{2}\langle 1 a \mid 1 b\rangle[(\mu 1 a \mid \nu 1 b)+(\mu 1 b \mid \nu 1 a)] \\
& +\frac{1}{2}[(\mu 1 b \mid 1 a 1 a)-(\mu 1 a \mid 1 a 1 b)]\langle 1 b \mid \nu\rangle \\
& +\frac{1}{2}\langle\mu \mid 1 b\rangle[(1 a 1 a \mid \nu 1 b)-(1 a 1 b \mid \nu 1 a)] .
\end{aligned}
$$

Again we can add terms to $H_{1 a}$ and $H_{2 a}$ to get an $H_{A}$ such that $\phi_{1 a}$ and $\phi_{2} a$ are both solutions of $H_{A}$ as in (4). For example, we would add

$$
\begin{aligned}
\Delta H{ }_{\mu \nu}^{2 a}= & \frac{1}{2}\langle 2 a \mid 1 b\rangle^{2}\langle\mu|h| \nu\rangle \\
& -\frac{1}{2}\langle\mu|h| 2 a\rangle\langle 2 a \mid 1 b\rangle\langle 1 b \mid \nu\rangle \\
& -\frac{1}{2}\langle\mu \mid 1 b\rangle\langle 1 b \mid 2 a\rangle\langle 2 a|h| \nu\rangle \\
& +\frac{1}{2}\langle\mu \mid 1 b\rangle\langle 1 b \mid \nu\rangle\langle 2 a|h| 2 a\rangle \\
& +(\mu \nu \mid 2 a 2 a)+(\mu \nu \mid 2 a 1 b)\langle 2 a \mid 1 b\rangle \\
& -(\mu 2 a \mid \nu 2 a) \\
& -\frac{1}{2}\langle 2 a \mid 1 b\rangle[(\mu 2 a \mid \nu 1 b)+(\mu 1 b \mid \nu 2 a)] \\
& +\frac{1}{2}[(\mu 1 b \mid 2 a 2 a)-(\mu 2 a \mid 2 a 1 b)]\langle 1 b \mid \nu\rangle \\
& +\frac{1}{2}\langle\mu \mid 1 b\rangle[(2 a 2 a \mid \nu 1 b)-(2 a 1 b \mid \nu 2 a)]
\end{aligned}
$$

to $H_{\mu \nu} 2 a$ to get $H_{\mu \nu}{ }^{A}$. (Note that $\sum_{\nu} \Delta H_{\mu \nu} 2 a$ $\times\langle\nu \mid 2 a\rangle=0$.) Just as in the UHF case, we find that the virtual solutions of $H_{2 a}$ are good approximations to the excited orbitals, whereas the virtual solutions to $H_{A}$ are not (see Table II).

Of course the above results apply equally well to other symmetries and other systems of various numbers of electrons. 


\section{DEPENDENCE OF PROPERTIES UPON EXCITED} STATES

The total electronic density at the nucleus, $\rho(0)$, is given in Table III for the various states of $\mathrm{Li}$. It is interesting to note that for $n s$ states the $\rho(0)$ decreases as we go to large $n$, eventually decreasing to the $\rho(0)$ of $\mathrm{Li}^{+}$, but that for $n p$ and $n d$ states $\rho(0)$ is less than the $\rho(0)$ of $\mathrm{Li}^{+}$and increases to $\rho(0)$ of $\mathrm{Li}^{+}$as $n$ and $l$ increase. ${ }^{24}$ An explanation of this behavior is that the valence electron, $n l$, partly shields the nucleus from the core electrons and thus leads to a slightly lower contribution to $\rho(0)$ from the core electrons, $\rho(0)$ core, as compared to $\mathrm{Li}^{+}$. If the added electron is in an $n s$ state, it has a nonzero amplitude at the nucleus which leads to an increase in $\rho(0)$ that more than cancels the previous decrease. But for $l \neq 0$ there is no compensating term, and $\rho(0)$ is lower for the atom than for $\mathrm{Li}^{+}$. (Note that $\rho(0)$ core is nearly independent of $l$ for $n s$ and $n p$ states.)

In addition, (see Table I) we have the order $2 s<2 p<3 s<3 p<3 d$ for total energy, whereas for $\left\langle\sum r^{2}\right\rangle$ we have the order $2 s<2 p<3 s<3 d<3 p$. Thus using $\left\langle\sum r^{2}\right\rangle$ as the criterion, $\phi_{3 d}$ is less diffuse than $\phi_{3 p}$, but more diffuse than $\phi 3 s$. However, for $\left\langle 1 / r^{3}\right\rangle_{n l}$ the order is $2 p<3 p<3 d$, which might be expected since the inner loop of the $3 p$ should count heavily here.

The value of $\left\langle 1 / r^{3}\right\rangle$ for various states $n l$ of the same $l$ are often ${ }^{25,26}$ expected to scale as $\left(1 / n^{*}\right)^{3}$, where $n^{*}$ is the effective quantum to use in the hydrogen atom formula in order to obtain the term energy. It has been less clear how the spin density term would scale, although it has also been assumed ${ }^{26}$ to scale as $\left(1 / n^{*}\right)^{3}$. In Table IV we compare the scaling of the hyperfine constants to that of $n^{*}$. We see that $\left(1 / n^{*}\right)^{3}$ leads to results too small by $1 \%$ and $8 \%$ for $Q(0)$ of the $3^{2} S$ and $3^{2} P$ states and too small by $2 \%, 4 \%$, and $13 \%$ for $\left\langle 1 / r^{3}\right\rangle$, $a_{1 / 2}$ and $a_{3 / 2}$ of the $3^{2} P$ state.

\section{CONCLUSIONS}

For the excited states of $\mathrm{Li}$ as for the ground state, the GF wave function leads to good values for the hyperfine parameters. Thus the major error in the Hartree-Fock value of the hyperfine splitting constants of $\mathrm{Li}$ is due to the lack of core polarization.
TABLE III. The density at the nucleus, $\rho(0)$, for $\mathrm{Li}^{+}$ and several states of $\mathrm{Li} . \rho(0)$ core is the density at the nucleus due to the $1 s$ electrons. The Hartree-Fock wave functions of Weiss (see Ref. 4) for $\mathrm{Li}$ and Roothaan, Sachs, and Weiss [Rev. Mod. Phys. 32,186 (1960)] for $\mathrm{Li}^{+}$were used.

\begin{tabular}{rcc}
\hline \hline & $\rho(0)$ & $\rho(0)$ core \\
\hline $\operatorname{Li} 2^{2} S$ & 13.816 & 13.652 \\
$3^{2} S$ & 13.708 & 13.666 \\
$2^{2} P$ & 13.653 & 13.653 \\
$3^{2} P$ & 13.666 & 13.666 \\
$3^{2} D$ & 13.672 & 13.672 \\
$\mathrm{Li}^{+} 1^{1} S$ & 13.674 & 13.674 \\
\hline
\end{tabular}

The calculated $a_{3 / 2}$ from GF and CI calculations disagree with the values deduced from the levelcrossing experiments. This casts doubt on the interpretation of these experiments in terms of effective $\left\langle 1 / r^{3}\right\rangle$ and $Q(0)$ that are independent of magnetic field.

The use of different Hamiltonians for different orbitals leads to improved convergence of excitedstate wave functions and also leads to virtual orbitals closely related to the orbitals of other excited states.

\section{APPENDIX A. THE CALCULATED WAVE FUNCTIONS}

The calculated orbitals for the UHF and GF wave functions are available upon request. The basis functions (which are Slater orbitals), the orbital energies $\left(\epsilon_{i}\right)$, and the amplitude at the nucleus $\left[\phi_{i}(0)\right]$ are given in Table $\mathrm{V}$.

\section{APPENDIX B: HYPERFINE SPLITTING CONSTANTS}

We define the hyperfine splitting constant, $A_{J}$, as the proportionality constant between $\overrightarrow{\mathrm{I}} \cdot \overrightarrow{\vec{J}}\rangle$ and the perturbation energy $E=A_{J}\langle\overrightarrow{\mathrm{I}} \cdot \overrightarrow{\mathrm{J}}\rangle$. Then we define $a_{J}$ by $A_{J}=g_{S} \mu_{B} g_{N} \mu_{N} a_{J}$, where $\mu_{B}$ and $\mu_{N}$ are the Bohr and nuclear magnetons, and $g_{s}$ and $g_{N}$ are the gyromagnetic ratios for the electron and nucleus. If the different states are all taken to be eigenstates of $\hat{L}^{2}$ and $\hat{S}^{2}$, then $a_{J}$ is ${ }^{27}$

TABLE IV. The scaling of the hyperfine constants for the $n s$ and $n p$ states of $\mathrm{Li}(n=2,3) . n^{*}$ is the effective quantum number obtained from the term energies (from Moore, footnote $\mathrm{c}$ to Table I).

\begin{tabular}{lcccccc}
\hline \hline & $n^{*}$ & $\left(1 / n^{*}\right)^{3}$ & $Q(0)$ & $\left\langle 1 / r^{3}\right\rangle$ & $a_{1 / 2}$ & $a_{3 / 2}$ \\
\hline $2 s$ & $1.588^{\mathrm{a}}$ & $\ldots$ & 0.2406 & $\ldots$ & $\ldots$ & $\ldots$ \\
$3 s$ & $2.596^{\mathrm{a}}$ & $\ldots$ & 0.05622 & $\ldots$ & $\ldots$ & $\ldots$ \\
ratio & $\ldots$ & 0.229 & 0.234 & $\ldots$ & $\ldots$ & $\ldots$ \\
$2 p$ & $1.966^{\mathrm{a}}$ & $\ldots$ & -0.02304 & 0.05861 & 0.2206 & -0.03313 \\
$3 p$ & $2.956^{\mathrm{a}}$ & $\ldots$ & -0.007318 & 0.01760 & 0.06735 & -0.01106 \\
ratio & $\ldots$ & 0.294 & 0.318 & 0.300 & 0.305 & 0.334 \\
\hline \hline
\end{tabular}


TABLE V. The orbital energy and amplitude at the nucleus from the UHF and GF wave functions of Li. All quantities are in Hartree atomic units. Basis sets: the entries represent the $n l \zeta$ of each Slater orbital (see Ref. 10).

\begin{tabular}{|c|c|c|c|c|c|c|c|}
\hline \multirow[b]{2}{*}{ State } & & \multicolumn{3}{|c|}{ Orbital Energy } & \multicolumn{3}{|c|}{ Amplitude at the Nucleus } \\
\hline & & $\epsilon_{1 a}$ & $\epsilon_{1 b}$ & $\epsilon_{2 a}$ & $\phi_{1} a^{(0)}$ & $\phi_{1 \mathrm{~b}}(0)$ & $\phi_{2 a}(0)$ \\
\hline \multirow[t]{2}{*}{$2^{2} S^{\mathrm{a}}$} & UHF & -2.48670 & -2.46873 & -0.19637 & 2.61848 & 2.60684 & -0.40486 \\
\hline & $\mathrm{GF}$ & -2.49875 & -2.46388 & -0.19649 & 2.63388 & 2.59173 & -0.40195 \\
\hline \multirow[t]{2}{*}{$3^{2} s^{\mathrm{a}}$} & UHF & -2.66484 & -2.66074 & -0.07380 & 2.61554 & 2.61287 & -0.19641 \\
\hline & GF & -2.66766 & -2.65952 & -0.07380 & 2.61922 & 2.60920 & -0.19612 \\
\hline \multirow[t]{2}{*}{$2^{2} P^{b}$} & UHF & -2.53133 & -2.53005 & -0.12868 & 2.61113 & 2.61448 & 0.00000 \\
\hline & $\mathrm{GF}$ & -2.53055 & -2.53220 & -0.12872 & 2.60614 & 2.61941 & 0.00000 \\
\hline \multirow[t]{2}{*}{$3^{2} P^{\mathrm{c}}$} & UHF & -2.67780 & -2.67735 & -0.05678 & 2.61349 & 2.61454 & 0.00000 \\
\hline & $\mathrm{GF}$ & -2.67755 & -2.67799 & -0.05678 & 2.61191 & 1.61611 & 0.00000 \\
\hline \multirow[t]{2}{*}{$3^{2} D^{\mathrm{d}}$} & UHF & -2.68123 & -2.68123 & -0.05556 & 2.61459 & 2.61460 & 0.00000 \\
\hline & GF & -2.68122 & -2.68124 & -0.05556 & 2.61458 & 2.61461 & 0.00000 \\
\hline
\end{tabular}

$\mathrm{a}_{1 s} 3.0,3 s 8.7,3 s 3.398,3 s 2.544,3 s 1.24,3 s 0.757,3 s 0.345,4 s 0.345$.

b1s $3.0,3 s 8.7,3 s 3.398,3 s 3.544,2 p 1.5,4 p 2.12,4 p 1.275,4 p 0.785,4 p 0.566$.

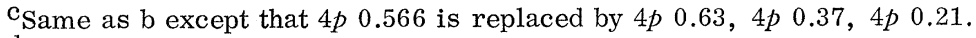

$\mathrm{d}_{s}$ orbtials as in $\mathrm{b}, 3 d 1.0,5 d 0.98,5 d 0.60,5 d 0.404,5 d 1.762$.

$$
\begin{aligned}
a_{J}= & \left(\frac{2}{g_{S}}\right) a_{l J(\overrightarrow{\mathrm{L}} \overrightarrow{\mathrm{J}}\rangle}+\frac{8 \pi}{3} a_{c} \frac{\langle\overrightarrow{\mathrm{S}} \cdot \overrightarrow{\mathrm{J}}\rangle}{J(J+1)} \\
& +a_{d} \frac{3\langle\overrightarrow{\mathrm{S}} \cdot \overrightarrow{\mathrm{L}}\rangle\langle\overrightarrow{\mathrm{L}} \cdot \vec{J}\rangle-L(L+1)\langle\overrightarrow{\mathrm{S}} \cdot \overrightarrow{\mathrm{J}}\rangle}{J(J+1)} .
\end{aligned}
$$

Relativistic effects and breakdown of $L S$ coupling have been ignored and $g_{l}=1$ has been assumed. The constants in the above equation are defined as

$$
\begin{aligned}
& a_{l}=\frac{1}{L}\left\langle L S L S\left|\sum_{e} \frac{\hat{l}_{z}}{r^{3}}\right| L S L S\right\rangle, \\
& a_{c}=\frac{1}{S}\left\langle L S L S\left|\sum_{e} \delta(r) \hat{s}_{z}\right| L S L S\right\rangle, \\
& a_{d}=\frac{1}{S L(2 L-1)}\left\langle L S L S\left|\sum_{e}\left(\frac{3 z^{2}-r^{2}}{r^{5}}\right) \hat{s}_{z}\right| L S L S\right\rangle, \\
& \langle\overrightarrow{\mathrm{L}} \cdot \overrightarrow{\mathrm{J}}\rangle \equiv \frac{1}{2}[J(J+1)+L(L+1)-S(S+1)], \\
& \overrightarrow{\mathrm{S}} \cdot J\rangle \equiv \frac{1}{2}[J(J+1)+S(S+1)-L(L+1)], \\
& \overrightarrow{\mathrm{S}} \cdot \overrightarrow{\mathrm{L}} \equiv \frac{1}{2}[J(J+1)-S(S+1)-L(L+1)],
\end{aligned}
$$

where $\left|L S M_{L} M_{S}\right\rangle$ denotes a many-electron wave function having quantum numbers $L, S, M_{L}$, and $M_{S}$. The subscripts $l, c$, and $d$ refer to orbital, Fermi contact, and spin-dipolar contributions, respectively.

For the HF, PUHF, and GF wave functions of Li, $a_{l}$ and $a_{d}$ simplify since

$$
\left\langle L S L S\left|\sum_{e} \frac{\hat{l}}{r^{3}}\right| L S L S\right\rangle=\left\langle\frac{1}{r^{3}}\right\rangle
$$

and

$$
\frac{1}{S}\left\langle\operatorname{LSLS}\left|\sum_{e} \frac{\left(3 z^{2}-r^{2}\right)}{r^{5}} \hat{s}_{z}\right| L S L S\right\rangle=\frac{-2 l}{(2 l+3)}\left\langle\frac{1}{r^{3}}\right\rangle,
$$

where $\left\langle 1 / r^{3}\right\rangle$ is a one-electron radial integral over the $l \neq 0$ orbital (with $m_{l}=l$ ).

Using the values ${ }^{28} g_{S}=2.0023192, \mu_{B}=9.2732$ $\times 10^{-24} \mathrm{~A} \mathrm{~m}^{2}, \mu_{N}=5.05050 \times 10^{-27} \mathrm{~A} \mathrm{~m}^{2}, 1 \mathrm{eV}$ $=1.60210 \times 10^{-19} \mathrm{~J}, 1 \mathrm{eV}=2.41804 \times 10^{14} \mathrm{~Hz}$, and $a_{0}$ $=0.529167 \AA$, we find that to convert $a_{J}$ from atomic units to $\mathrm{Mc} / \mathrm{sec}$ we have to multiply by $c=95.51975 g_{N}$. Thus for $\mathrm{Li}^{7}$ using ${ }^{29} g_{N}=3.25631 /$ 1.5, we obtain $c\left(\mathrm{Li}^{7}\right)=207.36_{1}$.

Using the above equations and letting $a_{c} \equiv Q(0)$ we obtain

for $n s^{2} S$ states: $a_{1 / 2}=8.3776 Q(0)$, for $n p^{2} P$ states: $a_{1 / 2}=2.6651\left\langle 1 / r^{3}\right\rangle-2.7925 Q(0)$,

$$
a_{3 / 2}=0.53256\left\langle 1 / r^{3}\right\rangle+2.7925 Q(0),
$$

for $n d^{2} D$ states: $a_{3 / 2}=0.99930\left\langle 1 / r^{3}\right\rangle-1.6755 Q(0)$,

$$
a_{5 / 2}=0.28525\left\langle 1 / r^{3}\right\rangle+1.6755 Q(0) \text {. }
$$

In the derivation it is necessary to assume that the wave functions are eigenfunctions of $L^{2}$ and $S^{2}$ and can be combined into eigenfunctions of $J^{2}$. This is correct for the HF, PUHF, and GF wave functions for $\mathrm{Li}$, but not for the UHF wave functions. Thus in the UHF case it is not correct to use the above equations (or any others for that matter) for $a_{J}$, since the UHF wave functions are not eigenfunctions of $S^{2}$ or $\mathrm{J}^{2}$. However, we will follow the usual practice of using the above equations for UHF also. 


\section{APPENDIX C: UPPER BOUNDS ON THE ENERGY FOR EXCITED STATES}

The UHF and GF wave functions for the excited states of Li lead to upper bounds on the exact energy, even though the wave functions are not orthogonal to the ground state. Below we sketch the proof of this for GF wave functions. ${ }^{30} \mathrm{~A}$ sim ilar theorem for $\mathrm{HF}$ wave functions has been proved using a different approach by Perkins. ${ }^{31}$

$$
\text { Let } \begin{aligned}
\psi_{1} & =G_{f}\left(\phi_{1 s}{ }^{a} \phi_{2 s}{ }^{a} \phi_{1 s}{ }^{b} \alpha \alpha \beta\right), \\
\psi_{2} & =G_{f}\left(\phi_{1 s}{ }^{a}{ }_{\phi_{3 s}} a_{\phi_{1 s}}{ }^{b} \alpha \alpha \beta\right),
\end{aligned}
$$

and consider linear combinations

$$
\psi=C_{1} \psi_{1}+C_{2} \psi_{2}
$$

of these functions. If the coefficients in (C-2) are chosen to make the total energy stationary, we find that the coefficients for the $i$ th solution satisfy

$$
\sum_{k}\left\langle\psi_{j}\left|H-E_{i}\right| \psi_{k}\right\rangle C_{j}=0 \text {. }
$$

In addition, from the Hylleraas-Undheim-Mac Donald theorem ${ }^{32}$ the calculated energy of the $i$ th solution is an upper bound on the exact energy for the $i$ th solution allowed by the symmetries of (C-1). But if the orbitals in (C-1) are solutions of the GF equations corresponding to (1), where $\phi_{1 s} a, \phi_{1 s}{ }^{b}$, and $\phi_{3 s} a$ are used in the field terms, then from the Brillouin theorem for GF wave functions, ${ }^{33}$ we have that

$$
\begin{aligned}
& \left\langle\phi_{1 s}{ }^{a} \phi_{c} \phi_{1 s}{ }^{b}\left|H-E_{2}\right| O_{f f} \phi_{1 s}{ }^{a} \phi_{3 s} a_{\phi_{1 s}}{ }^{b}\right\rangle \\
& =\left\langle\phi_{c}\left|H_{2 a}{ }^{G F}\right| \phi_{3 s} a^{a}\right\rangle=\epsilon_{3 s} a_{\left\langle\phi_{c} \mid \phi_{3 s}{ }^{a}\right\rangle=0}
\end{aligned}
$$

for any $\phi_{c}$ orthogonal to $\phi_{3 s}{ }^{a}$. Thus the solutions of $(\mathrm{C}-3)$ are $C_{1}=1, C_{2}=0$ and $C_{1}=0, C_{2}=1$; that is, the $\psi_{1}$ and $\psi_{2}$ in $(\mathrm{C}-1)$ are solutions of $(\mathrm{C}-3)$. But ${ }^{32}$

$$
\begin{aligned}
& E_{1}=\left\langle\psi_{1}|H| \psi_{1}\right\rangle /\left\langle\psi_{1} \mid \psi_{1}\right\rangle=E^{*}+\epsilon_{2 s}{ }^{a}, \\
& E_{2}=\left\langle\psi_{2}|H| \psi_{2}\right\rangle /\left\langle\psi_{2} \mid \psi_{2}\right\rangle=E^{*}+\epsilon_{3 s} a,
\end{aligned}
$$

where $E^{*}$ is the part of the energy term which depends only on $\phi_{1} a$ and $\phi_{1} b, \epsilon_{3 s} a$ is the eigenvalue for the occupied orbital $\phi_{3 s}{ }^{a}$, and $\epsilon_{2 s}{ }^{a}$ is the eigenvalue of the virtual orbital $\phi_{2 s} a$. Thus if $\epsilon_{2 s}{ }^{a}<\epsilon 3 s a$, then $E_{1}<E_{2}$ and $\psi_{2}$, the GF solution for the $3^{2} S$ state of $\mathrm{Li}$, yields an upper bound on the exact energy of the $3^{2} S$ state. This occurs despite the fact that $\psi_{2}$ is not orthogonal to the exact GF solution for the $2^{2} S$ state and a fortiori is not orthogonal to the exact solution of the $2^{2} S$ state.

Now consider the case where the $N$-electron wave function $\psi_{n}$ is a self-consistent GF solution of the $n$th state of a given symmetry and $\psi_{j}(j<n)$ are constructed by replacing $\phi_{n s} a$ by $\phi_{j s} a$, a lower energy virtual orbital of $\mathrm{H}_{2 a}$. Then (C-4) still holds for $\phi_{c}=\phi_{j s} a$ if $j \neq n$, and $\psi_{n}$ is already a solution of the equations corresponding to (C-3). Thus if there are $n-1$ virtual orbitals of lower energy, then $E_{n}=\left\langle\psi_{n}|H| \psi_{n}\right\rangle /\left\langle\psi_{n}|H| \psi_{n}\right\rangle$ is an upper bound on the energy of the $n$th state of this symmetry. This proof does not depend on the symmetry of the valence orbitals; it applies only to excited states obtained by exciting a single electron.

\footnotetext{
*Partially supported by a grant (GP-6965) from the National Science Foundation.

${ }^{\dagger}$ Alfred P. Sloan Research Fellow.

$\$$ Contribution No. 3703

${ }^{1}$ L. W. Anderson, F. M. Pipkin, and J. C. Baird, Phys. Rev. 116, 87 (1959).

${ }^{2}$ J. S. M. Harvey, Proc. Roy. Soc. (London) A285, 581 (1965).

${ }^{3}$ A. W. Weiss, Phys. Rev. 122, 1826 (1961).

${ }^{4}$ A. W. Weiss, Astrophys. J. 138, 1262 (1963).

${ }^{5}$ J. A. Pople and R. K. Nesbet, J. Chem. Phys. 22, 571 (1954); J. C. Slater, Phys. Rev. 82, 538 (1951); G. W. Pratt, Jr., Phys. Rev. 102, 1303 (1956); R. K. Nesbet, Proc. Roy. Soc. (London) A230, 312 (1955); A. T. Amos and G. G. Hall, ibid. $\underline{\underline{\mathrm{A} 263}}, 483$ (1961).

${ }^{6}$ P. O. Löwdin, Advan. Chem. Phys. 2, 207 (1959);

S. M. Blinder, Advan. Quant. Chem. 2, 47 (1965).

${ }^{7}$ W. A. Goddard, III, Phys. Rev. 157, 81 (1967).

${ }^{8}$ W. A. Goddard, III, Phys. Rev. 157, 93 (1967).

${ }^{9}$ W. A. Goddard, III, J. Chem. Phys. 48, 450 (1968).

${ }^{10} \mathrm{~A}$ Slater orbital is a function of the form $\chi_{n l m}$ $=N_{n} r^{n-1} e^{-\zeta r} Y_{l m}$, where $\zeta$ is a variable parameter called the orbital exponent and $N_{n}$ is a normalization factor.
}

\footnotetext{
${ }^{11}$ For the $2{ }^{2} S$ state of $\mathrm{Li}$ the CI calculations quoted (Ref. 12) used interelectronic coordinates. The best CI calculation on this state not using interelectronic coordinates is that of Weiss (Ref. 3) which yielded a poor value (Ref. 13) for $Q(0)$ of 0.2065 [compared to the experimental value of 0.2313 (Ref. 14) ]. The reason for this poor $Q(0)$ is that Weiss did not include any configurations combining $s$-type core orbitals into a triplet. It is that such configurations which are needed for the core orbitals to contribute to $Q(0)$. The CI calculation on the $2^{2} P$ state (Weiss, Ref. 4) did include such configurations, and thus can be expected to yield a more reliable value for $Q(0)$. However, it is possible that an insufficient number of such configurations was used; in this case the CI value of $Q(0)$ may not have converged to the correct value.

${ }^{12}$ G. J. Ritter, Canadian J. Phys. 43, 770 (1965).

${ }^{13}$ K. C. Brog, T. G. Eck, and H. Wieder, Phys. Rev. 153,91 (1967).

${ }^{14}$ In order to interpret the level crossing experiments in terms of $\left\langle 1 / \gamma^{3}\right\rangle$ and $Q(0)$, Brog et al. (Ref. 16) allowed a general mixing of zero-field configurations by the external field. But they allowed only the six zero-field configurations appropriate for $\mathrm{Li} 2{ }^{2} P$, and assumed that these were related to each other and to
} 
$\left\langle 1 / r^{3}\right\rangle$ and $Q(0)$ as they would be for Hartree-Fock wave functions.

${ }^{15}$ R. W. B. Ardill and A. L. Stewart, J. Chem. Phys. 47, 4853 (1967); Proc. Phys. Soc. (London) 92, 296 (1967).

${ }^{16}$ D. A. Goodings, Phys. Rev. 123, 1706 (1961).

${ }^{17}$ M. H. Cohen, D. A. Goodings, and V. Heine, Proc. Phys. Soc. (London) $\underline{73}, 811$ (1959).

${ }^{18}$ G. D. Gaspari, W. M. Shyu, and T. P. Das, Phys. Rev. 134, A852 (1964).

${ }^{19}$ W. Marshall, J. Phys. Soc. Japan 17, Suppl. B-120 (1962) .

${ }^{20}$ Burke (Ref. 12b) has also concluded that the concept of core polarization by non-s electrons is valid, but Bergreen and Wood [Phys. Rev. 130, 198 (1963)] question the idea of core polarization by $s$ electrons.

${ }^{21}$ J. B. Martin and A. W. Weiss, J. Chem. Phys. 39, 1618 (1963).

${ }^{22}$ T. Koopmans, Physica 1, 104 (1933).

${ }^{23}$ Here the orbitals are taken to be real, $\langle 1 a \mid 1 b\rangle$ denotes an overlap integral, $\langle 1 a|h| 1 b\rangle$ denotes a oneelectron integral, and

$$
\begin{aligned}
(\alpha \beta \mid \gamma \delta)= & \int d \overrightarrow{\mathrm{x}}_{1} \phi_{\alpha}{ }^{*(1) \phi_{\beta}(1)} \\
& \times \int d \overrightarrow{\mathrm{x}}_{2}\left(1 / r_{12}\right) \phi_{\gamma}{ }^{*(2) \phi_{\delta}}(\gamma)
\end{aligned}
$$

is the usual notation for a two-electron integral. We have multiplied (4) by $\left(1+\frac{1}{2}\langle 1 a \mid 1 b\rangle^{2}\right)$ for convenience

${ }^{24}$ Comparing the $\rho(0)$ for the $\mathrm{HF}$ wave function of $\mathrm{Li}^{+}$ to the value for the very accurate Pekeris wave function [Phys. Rev. 126, $143(1962)$ ], $\rho(0)=13.704$, we would expect the $\rho(0)$ for the various states of $\mathrm{Li}$ in Table II to also be lower than the exact values by about 0.03 or $0.2 \%$.

${ }^{25}$ R. Isler, S. Marcus, and R. Novick, Bull. Am. Phys. Soc. 11, 62 (1966).

${ }^{26}$ B. Budick, H. Bucka, R. J. Goshen, A. Landman, and R. Novick, Phys. Rev. 147, 1 (1966).

${ }^{27}$ For example, see R. E. Trees, Phys. Rev. 92, 308 (1953).

${ }^{28}$ E. R. Cohen and J. W. M. Dumond, Rev. Mod. Phys. 37, 537 (1965).

${ }^{29}$ N. F. Ramsey, Molecular Beams (Oxford, Clarendon Press, London, 1956) p. 172.

${ }^{30}$ The basic idea of using a configuration interaction approach to prove this theorem was suggested by Professor R. M. Pitzer, private communication, 1966.

${ }^{31}$ J. F. Perkins, J. Chem. Phys. 42, 3927 (1965).

${ }^{32} \mathrm{E}$. Hylleraas and B. Undheim, Z. Physik 65,759 (1930); J. K. L. MacDonald, Phys. Rev. $43, \overline{830}$ (1933).

${ }^{33}$ W. A. Goddard, III, J. Chem. Phys. 48,5337 (1968).

\title{
Relativistic Calculations of Electron Binding Energies by a Modified Hartree-Fock-Slater Method
}

\author{
Arne Rosen and Ingvar Lindgren \\ Department of Physics, Chalmers University of Technology, Göteborg, Sweden \\ (Received 8 July 1968)
}

\begin{abstract}
Relativistic atomic wave functions and electron binding energies have been calculated by a modified Hartree-Fock-Slater method. The local exchange approximation, originally introduced by Slater, is modified in such a way that the total energy of the system is minimized. Approximate expressions for the optimum exchange potential are suggested for all atoms and ions. The total atomic energies and electron binding energies obtained by this method agree extremely well with the corresponding Hartree-Fock results in the cases where such data are available. Various corrections to the theoretical binding energies are discussed, in particular the effect of rearrangement during the ionization process. It is found that this effect is of importance for inner shells in all elements and is responsible for the main discrepancy between experimental and previous theoretical results for light and medium-heavy elements. For heavy elements other effects are of importance, and various possible sources of the residual discrepancy between theory and experiments are discussed.
\end{abstract}

\section{INTRODUCTION}

During the last decade our knowledge of electron binding energies in atoms has increased considerably, due in particular to the development of the electron-spectroscopic method (ESCA) by Siegbahn et $a l_{\infty}{ }^{1}$ This method has been applied to almost all elements between lithium and berkelium and to inner as well as outer shells. The accuracy of the new data is so high that improved theoretical calculations are justified. The electron binding energies are often used to check the accuracy of electronic wave functions. When the accuracy of the calculations increases, several effects have to be taken into account. Besides the relativistic effects, which of course are dominant for heavy elements, it is necessary to consider relaxation mechanisms, correlation effects, core polariza- 